\title{
Functional Outcome of Transphyseal Reconstruction of the Anterior Cruciate Ligament in Skeletally Immature Patients
}

\author{
Sung-Jae Kim, $\mathrm{MD}, \mathrm{PhD}^{1}$, Dong-Woo Shim, $\mathrm{MD}^{1}$ and Kwang-Won Park, $\mathrm{MD}^{2}$ \\ ${ }^{1}$ Department of Orthopaedic Surgery, Severance Hospital, Yonsei University College of Medicine; ${ }^{2}$ Institute for Rare Diseases and Department of Orthopaedic Surgery, \\ Guro Hospital, Korea University Medical Center, Seoul, Korea
}

\begin{abstract}
Purpose: In anterior cruciate ligament (ACL) injury, conventional adult reconstruction techniques have to face the potential risk of growth disturbance or angular deformities in skeletally immature patients. The aim of this study was to evaluate the clinical outcomes of ACL reconstruction by conventional transphyseal tunnel technique.

Materials and Methods: On a retrospective basis, we reviewed 25 skeletally immature patients; all the patients showed skeletal maturity at last followup, and the mean age was 16.4 years. The average injury to surgery interval was 12.6 months. Clinical outcomes were assessed at a mean of 74.4 months postoperatively using the Lysholm Knee Scoring Scale, the Tegner activity level, the International Knee Documentation Committee (IKDC), and plain radiographs.

Results: All the patients had undergone transphyseal reconstruction of ACL. The mean Lysholm score was 48.36 points preoperatively and 93.32 points postoperatively; the mean Tegner activity level was changed from 3.0 points to 5.6 points. The mean IKDC level was categorized as C preoperatively and changed to A postoperatively.

Conclusions: Our midterm outcome at an average 6 years after surgery was satisfactory without significant leg length discrepancies or abnormal alignment of the knee joint. Transphyseal reconstruction of ACL is a good treatment modality in the skeletally immature patient.
\end{abstract}

Key words: Anterior cruciate ligament, Skeletally immature, Adolescent.

\section{Introduction}

Knee injuries, particularly anterior cruciate ligament (ACL) tears, in skeletally immature patients have been increasing due to an increase in the participation of adolescents in sports and leisure activities ${ }^{1)}$. There has been much debate over whether

Received September 20, 2011; Revised (1st) October 17, 2011;

(2nd) December 4, 2011; (3rd) July 2, 2012; Accepted July 3, 2012.

Correspondence to: Kwang-Won Park, MD.

Institute for Rare Diseases and Department of Orthopaedic Surgery, Guro Hospital, Korea University Medical Center, 148 Gurodong-ro, Guro-gu, Seoul 152-703, Korea.

Tel: +82-2-2626-1163, Fax: +82-2-2626-1164

Email:kwpark77@gmail.com

This is an Open Access article distributed under the terms of the Creative Commons Attribution Non-Commercial License (http://creativecommons.org/licenses/by-nc/3.0/) which permits unrestricted non-commercial use, distribution, and reproduction in any medium, provided the original work is properly cited. conservative or surgical treatment is better for ACL tears in skeletally immature patients with growth potential. Recently, surgical intervention is recommended to avoid the complications of conservative treatment, which include additional meniscal tears or early onset of degenerative arthritis ${ }^{2-4)}$.

There are a variety of ACL reconstruction techniques that allow for minimal physeal damage. When it comes to transphyseal ACL reconstruction, one of the most important criteria to determine the timing of surgery is the amount of remaining growth potential, because physeal damage during the procedure can lead to premature growth plate closure, growth disturbances, leg length discrepancies, or angular deformities ${ }^{5-9)}$.

The timing of growth plate closure can be different for each patient and skeletal maturity should be assessed by taking various factors into consideration including Tanner stage, Risser sign, adolescent growth spurt, and comparison of the patient's height to that of the other family members. In practice, skeletal maturity is determined by radiographic evidence of physeal closure. Proper treatment selection for relatively young patients can be made without difficulty if skeletal maturity is considered achieved 
based on radiographic evidence of physeal closure. In contrast, utmost care should be taken in determining an appropriate treatment timing and method in late adolescents (around the age of 15 years) with skeletal immaturity.

In this study, we performed ACL reconstruction in skeletally immature patients and evaluated the long-term functional and radiological outcomes of the surgery at the time of skeletal maturity.

\section{Materials and Methods}

A total of 158 patients with $\leq 18$ years of age underwent transphyseal ACL reconstruction at our institution between January 1993 and December 2008. Of these patients, 25 patients (25 cases) who were available for $\geq 2$ years of follow-up and showed skeletal maturity at last follow-up were reviewed retrospectively for this study. From each patient's medical records the following were assessed: the mechanism of injury, injury to surgery interval, preoperative clinical examination findings, preoperative knee function (Lysholm score), preoperative sports participation level (Tegner activity level), and presence of a combined injury. Leg length discrepancy and abnormal knee alignment were evaluated on radiographs. It was difficult to obtain a precise Tanner staging based on retrospective review of medical records and radiographs. Therefore, the presence of a gap between the growth plates on anteroposterior (AP) radiographs was considered evidence of skeletal immaturity (Fig. 1). Knees with a patellar ligament injury that required surgery or posterolateral instability were excluded from the study. There were 15 boys with a mean age of 16 years and 4 months (range, 9 years and 8 months 17 years and 9 months) and 10 girls with a mean age of 16 years and 6 months (range, 14 years and 2 months 17 years and 9 months).

The combined injuries were medial meniscal tear in 4 cases, lateral meniscal tear in 8 cases, and bilateral meniscal tears in 1 case. The mechanism of injury was sports related in 24 cases (soccer in 10 cases, basketball in 9 cases, cycling in 1 case, horse vault in 2 cases, judo in 1 case, and ski in 1 case) and falling in 1 case. The mean injury to surgery interval was 12.6 months (range, 1-40 months).

ACL reconstruction was performed using a transphyseal tunnel technique in all patients. Care was taken to minimize the bone tunnel size relative to the entire physes during tunnel placement. Taking care not to damage the tibial physis, tibial tunnel drilling was started medial to the tibial spine at an angle of $<30^{\circ}$ from the tibial axis toward the tibial isometric point. A femoral tunnel was drilled at an angle of $45^{\circ}$ from the femoral physis in the over-the top' position using a reamer that was rotated counterclockwise to prevent additional damage to the physis. The drill holes were $<8 \mathrm{~mm}$ in diameter (Fig. 2). A tendon graft was placed in the tunnel to prevent bony bridge formation. Femoral graft fixation was done using the Endobutton (Smith \& Nephew, Andober, MA, USA). Tibial graft fixation was achieved using either a screw if the tunnel length below the physis was sufficient $(\geq 30 \mathrm{~mm}$ ) or an endowasher or a buckle staple if the tunnel length was 30 $\mathrm{mm}$. The reconstruction was performed using a single-bundle technique in all patients. The graft used was hamstring tendon autograft in 22 cases and tibialis posterior allograft in 3 cases.

On the postoperative clinical assessment, generalized ligamentous laxity was assessed, the Lachman test, pivot shift test, and drawer sign test were performed at the last follow-up evaluation, and objective assessment was carried out using a KT


Fig. 1. (A) Initial radiographic image of 14 years 7 months old male patient shows wide opened physis on femoral and tibial side. The patient injured his left knee as a result of falling down from a desk. (B, C) Preoperative magnetic resonance imaging image shows intrasubstance tear of anterior cruciate ligament. 
1000/2000 arthrometer (MEDmetric, San Diego, CA, USA). All the clinical evaluations were performed by two orthopedic surgeons. The clinical results were evaluated according to the International Knee Documentation Committee (IKDC) criteria. The last follow-up radiographs taken at skeletal maturity were assessed to identify early growth plate closure, leg length discrepancies, angular deformities, early degenerative changes, and side-to-side differences. The Lysholm score was used to assess the improvement in clinical function and the Tegner activity level to evaluate the level of return to sports activities.

Statistical analysis was performed using SPSS ver. 18.0 (SPSS Inc., Chicago, IL, USA). The Wilcoxon-sign rank test was used to compare the preoperative and postoperative knee function. The Mann-Whitney $\mathrm{U}$ test was used to compare the radiographic measurements of the operated and non-operated sides at the last follow-up evaluation.

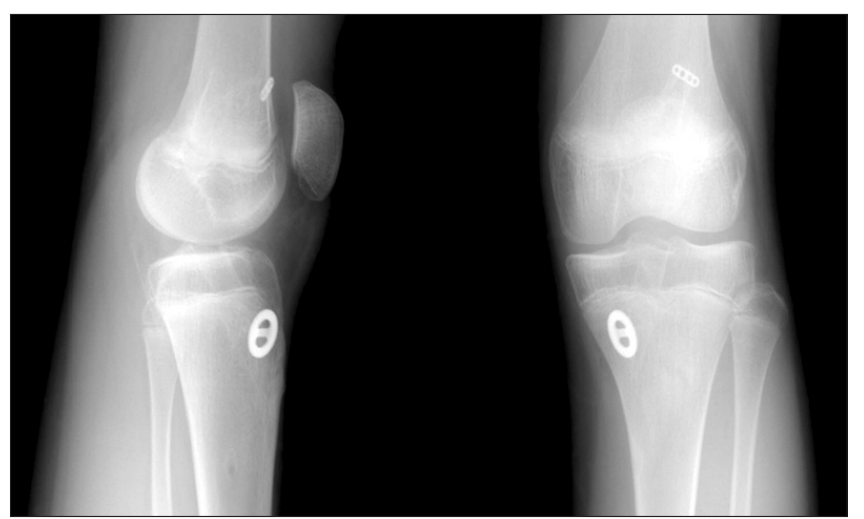

Fig. 2. Postoperative 2 years plain radiographs shows still clearly open physis, but no deterioration of epiphyseal shape.

\section{Results}

The mean follow-up period was 74.4 months (range, 25-216 months). The mean age of the patients at last follow-up was 22 years and 7 months (range, 17 year -36 years and 3 months). Of the boys, 3 patients who did not appear to have gone through adolescent growth spurt were classified as Tanner stage 3 and the remaining 12 patients, as Tanner stage 4 . All the girls $(n=10)$ including the two whose first menstruation started within 1 year after surgery were classified as Tanner stage 4 . The possibility of Tanner stage 5 was ruled out because there was no radiographic evidence of growth plate closure and $\geq 3 \mathrm{~cm}$ leg length growth was observed after surgery in all of them.

On the preoperative knee function, the IKDC level was categorized as C (68\%) in most of the cases: A in 1 case (4\%), B in 2 cases (8\%), $C$ in 17 cases (68\%), and D in 5 cases (20\%). The IKDC level was improved overall postoperatively: A in 22 cases $(88 \%)$ and B in 3 cases (12\%) (Table 1). The mean KT 1000/2000 arthrometer side-to-side difference was improved from 10.6 $\mathrm{mm}$ (range, 9-14 $\mathrm{mm}$ ) preoperatively to $3.4 \mathrm{~mm}$ (range, $2-8$ $\mathrm{mm})$ postoperatively $(\mathrm{p}<0.001)$. The mean Lysholm score was improved from 48.8 (range, 5-92) preoperatively to 93.3 (range, 79-100) at last follow-up $(\mathrm{p}<0.001)$. The mean Tegner activity

Table 1. Data of Functional Results

\begin{tabular}{lcc}
\hline & Preoperative data & Last follow-up \\
\hline IKDC & C & A \\
KT 1000/2000 arthrometer ${ }^{\text {a) }}$ & $10.56(9-14)$ & $3.36(2-8)$ \\
Lysholm score $^{\text {a) }}$ & $48.36(5-92)$ & $93.32(79-100)$ \\
Tegner activity level $^{\text {a) }}$ & $3.0(1-7)$ & $5.6(4-10)$ \\
\hline
\end{tabular}

IKDC: International Knee Documentation Committee.

${ }^{a)}$ Significant adjusted p-value $<0.05$.

Table 2. Radiographic Outcomes Compared with Contralateral Uninjured Knee

\begin{tabular}{|c|c|c|c|c|}
\hline \multirow{2}{*}{ Mean value } & \multicolumn{2}{|c|}{ Initial } & \multicolumn{2}{|c|}{ Last follow-up } \\
\hline & Ipsilat & Contralat & Ipsilat & Contralat \\
\hline mLDFA $\left(^{\circ}\right)(\mathrm{NL}: 85-90)$ & $86.8(85.0-91.0)$ & $87.0(84.5-89.0)$ & $87.0(83.0-90.0)$ & $88.0(84.0-90.0)$ \\
\hline $\operatorname{mMPTA}\left({ }^{\circ}\right)(\mathrm{NL}: 85-90)$ & $88.5(86.0-91.0)$ & $88.1(85.0-90.0)$ & $88.0(87.0-90.0)$ & $88.1(86.5-90.0)$ \\
\hline F-T angle $\left({ }^{\circ}\right)$ & $6.0(4.0-7.0)$ & $6.1(5.0-7.0)$ & $6.1(5.0-7.0)$ & $6.2(5.0-8.0)$ \\
\hline Entire leg length $(\mathrm{cm})$ & $79.5(70.8-87.1)$ & $79.4(70.5-86.9)$ & $84.1(75.2-93.2)$ & $84.1(74.9-93.4)$ \\
\hline $\mathrm{PDFA}^{\text {a) }}$ (NL: 79-87) & $83.36(80.0-87.0)$ & $82.36(78.0-87.0)$ & $83.44(81.0-86.0)$ & $84.04(81.0-87.0)$ \\
\hline PPTA $^{\text {a) }}$ (NL: 77-85) & $81.48(79.0-84.0)$ & $81.2(79.0-85.0)$ & $81.92(79.0-84.0)$ & $82.04(80.0-85.0)$ \\
\hline
\end{tabular}

Ipsilat: ipsilateral, Contralat: contra lateral, mLDFA: mechanical lateral distal femoral angle, NL: normal, mMPTA: mechanical medial proximal tibial angle, F-T angle: femorotibial angle, PDFA: posterior distal femoral angle, PPTA: posterior proximal tibial angle.

${ }^{\text {a) }}$ Significant adjusted p-value $<0.05$. 




Fig. 3. Postoperative 8 years scanogram of the lower extremity shows no axial deviation or discrepancies of leg length.

level was improved from 3.0 (range, 1-7) preoperatively to 5.6 (range, 4-10) postoperatively $(\mathrm{p}<0.001)$. The mean amount of leg length growth from the time of surgery to last follow-up was 4.6 $\mathrm{cm}$ (range, 3.1-9.6 cm) on the operated side and $4.7 \mathrm{~cm}$ (range, 2.9-9.6 cm) on the non-operated side (Table 2). There was no observed leg length discrepancy of $\geq 2.5 \mathrm{~cm}$ in any of the patients. The side-to-side difference was $<1 \mathrm{~cm}$ in most of the patients ( 21 cases, $84 \%$ ). Although $\geq 1 \mathrm{~cm}$ side-to-side difference was noted in 4 cases (range, 1.1-1.6), it was not clinically significant. There was no observed angular deformity of $\geq 5^{\circ}$ in any of the patients on the coronal and sagittal views (Fig. 3). Albeit within the normal range, the posterior distal femoral angle on the sagittal plane decreased significantly after surgery on the operated side compared to the preoperative measurement $(\mathrm{p}=0.046)$ and to the non-operated side $(\mathrm{p}=0.002)$. Posterior proximal tibial angle was not significantly changed between the preoperative and postoperative period $(\mathrm{p}=0.137)$, but decreased on the operated side compared to the non-operated side $(\mathrm{p}=0.025)$ indicating a tendency to an increase in the posterior tibial slope. Degenerative arthritis combined with subchondral sclerosis was observed on the radiograph in 1 patient after reconstruction using a hamstring tendon autograft. The patient was a professional basketball player and had generalized ligamentous laxity and knee instability. Surgery-related complications, such as infections, were not observed in all the patients and $10^{\circ}$ of flexion contracture was noted in 1 case.

\section{Discussion}

The treatment of choice for ACL tears in adolescents should be determined with care according to the stage of skeletal maturity. Based on the magnetic resonance imaging evaluation of the closure of distal femoral and proximal tibial physes, Sasaki et al. ${ }^{10}$ reported that skeletal maturity is reached by the age of 16 in boys and 14 in girls. However, skeletal maturity cannot be assessed solely by age, but is considered achieved in general based on the following criteria: $\geq$ Tanner stage 4 or $5,<2.5 \mathrm{~cm}$ difference in height with other family members, adolescent growth spurt, and radiographic evidence of growth plate closure ${ }^{2,11}$. ACL tears in adolescents can be reconstructed after skeletal maturity or growth spurt with the same method used in adults. On the other hand, utmost care has been taken in the determination of treatment timing and methods in patients who are skeletally immature for their chronological age, as in our study, to avoid possible complications of growth plate damage.

ACL tears in skeletally immature patients can be treated either non-surgically or surgically with primary repair, physeal sparing reconstruction, partial physeal sparing reconstruction, or transphyseal reconstruction. The recent consensus is that surgical management of ACL tears is more advantageous over conservative treatments, considering that the latter has been associated with meniscal damage and knee instability, which can cause additional ligament damage and early onset of degenerative arthritis in the long term ${ }^{3,12-14)}$. However, the established reconstruction techniques using femoral and tibial tunnels carry the risk of growth plate damage, leg length discrepancies, or abnormal knee alignment for patients with open physes ${ }^{15-18)}$. Thus, a variety of attempts have been made to prevent or to minimize the potential side effects.

Janarv et al. ${ }^{4)}$ reported that growth retardation occurred when $7-9 \%$ of the distal femoral physis was destroyed, whereas retardation was not observed when of $4-5 \%$ of the physis was injured in a rabbit model. In the study, bony bridge formation could be prevented with the placement of a tendon graft in a transphyseal tunnel. Stadelmaier et al. ${ }^{19)}$ reported that a fascia lata autograft placed in tunnels drilled across growth plates prevented formation of a bony bridge in a canine model. Minimal physeal injury and graft placement in bone tunnels have been advocated in many later studies as well. Bales et al. ${ }^{8)}$ suggested the use of relatively small 7-8 $\mathrm{mm}$ bone tunnels, the placement of a graft within the tunnels, and the performance of fixation proximal to the physis in the femur and distal to the physis in the tibia. According to the 3D CT study by Shea et al. ${ }^{20}$, proximal tibial 
tunnel that started more medial, distal, and with a steeper angle of inclination, significantly reduced damage to the tibial physis.

In the present study, transphyseal ACL reconstruction was performed with care to minimize damage to the femoral and tibial physes in all patients, as suggested in the abovementioned studies. A hamstring tendon autograft or a posterior tibialis allograft was placed within bone tunnels. The tibial bone tunnel was placed as close as possible to the center of the physis with an inclination of $30^{\circ}$ with regard to the tibial axis to be perpendicular to the tibial physis. The femoral bone tunnel was created with an inclination of $45^{\circ}$ to avoid excessive damage to the physes. The diameter of the bone tunnels was $<7-8 \mathrm{~mm}$, which was $<5 \%$ of the total area of the physes and accordingly resulted in reduced complication rates. Femoral graft fixation was performed proximal to the physis using the Endobutton (femoral cortical suspensory fixation). Tibial fixation was achieved distal to the physis by a screw if the tunnel was $\geq 30 \mathrm{~mm}$ in length and by an endowasher or a buckle staple for a $<30 \mathrm{~mm}$ tunnel.

Surgical techniques designed to minimize the risk of physeal injury have produced relatively successful results in many studies. Unfortunately, those techniques have not been tested in a large number of subjects and are technically challenging ${ }^{21-24)}$. Considering that the prevalence of complications of physeal injury is very low $^{15-18)}$ and experienced surgeons produce more satisfactory results compared to the inexperienced ${ }^{25)}$, we believe that transphyseal reconstruction is a relatively safe and promising surgical procedure for ACL tears.

Streich et al. ${ }^{26)}$ reported successful clinical results of transphyseal ACL reconstruction in 17 patients with Tanner stage 1 or 2 . There were no leg length discrepancies or abnormal knee alignments in any of the patients. Frosch et al. ${ }^{27)}$ performed a meta-analysis to compare various ACL reconstruction techniques for skeletally immature patients. According to the study, the incidence of leg length discrepancies or abnormal knee alignment after ACL reconstruction was relative low and physeal sparing techniques resulted in higher complication rates.

The posterior distal femoral angle of the reconstructed side on the sagittal plane decreased postoperatively in our study. This can be attributed to the femoral physeal injury caused by the $45^{\circ}$ inclination of the femoral tunnel with regard to the tibia. The decrease in the posterior proximal tibial angle that resulted in the increase in the posterior tibial slope of the reconstructed side was also attributed to unavoidable physeal injury. However, all the values were within the normal range. In addition, side-toside differences in the sagittal plane alignment would not have significant influence on the clinical knee function due to the compensatory mechanism compared to those in the coronal plane alignment ${ }^{28)}$. The one patient with flexion contracture showed no signs of abnormal sagittal plane alignment including distal femoral flexion, an increase in the proximal tibial posterior slope, or displacement of the anatomical axis from the preoperative to the last follow-up period. Therefore, we thought that the $10^{\circ}$ flexion contracture was caused by soft tissue contracture that occurred during the postoperative immobilization period.

In a study by Briggs et al. ${ }^{29)}$, the mean Lysholm score was 94 and the mean Tegner activity level was 5.7 in 488 normal adult subjects. In our study, the mean Lysholm score was 93.32 and the mean Tegner activity level was 5.6 at the last follow-up, which shows that our values were similar to those found in normal adults.

In the patient who showed knee joint instability after surgery, generalized ligament laxity was present and reconstruction was carried out using a hamstring tendon autograft. Kim et al. ${ }^{30}$ reported that the results of reconstruction using autologous bone-patellar tendon-bone grafts were better than those using hamstring grafts in patients with generalized ligament laxity. However, reconstruction using an autologous bone-patellar tendon-bone graft is avoided in general in skeletally immature patients because of the risk of physeal injury in the graft donor site or remaining bone tissues in the bone tunnel. The use of autologous hamstring tendon grafts in patients with open physes can be advantageous in that donor site physeal injury can be prevented and fixation can be achieved away from the physes by placing a sufficient length of graft in the bone tunnel. However, we had difficulty in associating the cause of instability with the graft choice in this study because the patient was a professional athlete.

Except for the 3 patients in Tanner stage 3, 22 patients showed Tanner stage 4 of sexual maturity. Physeal closure did not occur in them despite the chronological age and the mean $4 \mathrm{~cm}$ leg length growth was observed until the last follow-up. This indicates that the possibility of constitutional growth delay should also be considered prior to treatment selection. Thus, the level of skeletal maturity should be determined by considering various factors based on thorough clinical and radiographic assessments. The growth pattern and illness history of the patient's family, level of daily living activities, and sports participation level should also be assessed prior to determining surgical techniques and selecting grafts.

The limitations of this study include the retrospective design, relatively small number of patients, relatively old age of the 
patients with a mean age of 16 years, and the use of 2 different grafts. In addition, the possible long-term effects of the sideto-side difference in the sagittal plane alignment were not thoroughly identified. The significance of this study is that it showed the efficacy of transphyseal ACL reconstruction for skeletally immature patients based on the long-term follow-up evaluations that were performed after the patient's had achieved skeletal maturity.

\section{Conclusions}

We believe that transphyseal reconstruction produces satisfactory results for the treatment of ACL tears in adolescents with open physes without the risk of leg length discrepancies or abnormal alignment in the coronal plane.

\section{References}

1. Aronowitz ER, Ganley TJ, Goode JR, Gregg JR, Meyer JS. Anterior cruciate ligament reconstruction in adolescents with open physes. Am J Sports Med. 2000;28:168-75.

2. McCarroll JR, Rettig AC, Shelbourne KD. Anterior cruciate ligament injuries in the young athlete with open physes. Am J Sports Med. 1988;16:44-7.

3. Mizuta H, Kubota K, Shiraishi M, Otsuka Y, Nagamoto $\mathrm{N}$, Takagi $\mathrm{K}$. The conservative treatment of complete tears of the anterior cruciate ligament in skeletally immature patients. J Bone Joint Surg Br. 1995;77:890-4.

4. Janarv PM, Wikstrom B, Hirsch G. The influence of transphyseal drilling and tendon grafting on bone growth: an experimental study in the rabbit. J Pediatr Orthop. 1998; 18:149-54.

5. Aichroth PM, Patel DV, Zorrilla P. The natural history and treatment of rupture of the anterior cruciate ligament in children and adolescents. A prospective review. J Bone Joint Surg Br. 2002;84:38-41.

6. Johnston DR, Ganley TJ, Flynn JM, Gregg JR. Anterior cruciate ligament injuries in skeletally immature patients. Orthopedics. 2002;25:864-71.

7. Shea KG, Apel PJ, Pfeiffer RP. Anterior cruciate ligament injury in paediatric and adolescent patients: a review of basic science and clinical research. Sports Med. 2003;33:455-71.

8. Bales CP, Guettler JH, Moorman CT 3rd. Anterior cruciate ligament injuries in children with open physes: evolving strategies of treatment. Am J Sports Med. 2004;32:1978-85.

9. Kaeding CC, Flanigan D, Donaldson C. Surgical techniques and outcomes after anterior cruciate ligament reconstruction in preadolescent patients. Arthroscopy. 2010;26:1530-8.

10. Sasaki T, Ishibashi Y, Okamura Y, Toh S. MRI evaluation of growth plate closure rate and pattern in the normal knee joint. J Knee Surg. 2002;15:72-6.

11. Tanner JM. Growth at adolescence: with a general consideration of the effects of hereditary and environmental factors upon growth and maturation from birth to maturity. 2nd ed. Oxford: Blackwell Scientific; 1962.

12. Angel KR, Hall DJ. Anterior cruciate ligament injury in children and adolescents. Arthroscopy. 1989;5:197-200.

13. Graf BK, Lange RH, Fujisaki CK, Landry GL, Saluja RK. Anterior cruciate ligament tears in skeletally immature patients: meniscal pathology at presentation and after attempted conservative treatment. Arthroscopy. 1992;8:22933.

14. Parker AW, Drez D Jr, Cooper JL. Anterior cruciate ligament injuries in patients with open physes. Am J Sports Med. 1994;22:44-7.

15. Lipscomb AB, Anderson AF. Tears of the anterior cruciate ligament in adolescents. J Bone Joint Surg Am. 1986;68:1928.

16. Nakhostine M, Bollen SR, Cross MJ. Reconstruction of midsubstance anterior cruciate rupture in adolescents with open physes. J Pediatr Orthop. 1995;15:286-7.

17. Koman JD, Sanders JO. Valgus deformity after reconstruction of the anterior cruciate ligament in a skeletally immature patient. A case report. J Bone Joint Surg Am. 1999;81:711-5.

18. McIntosh AL, Dahm DL, Stuart MJ. Anterior cruciate ligament reconstruction in the skeletally immature patient. Arthroscopy. 2006;22:1325-30.

19. Stadelmaier DM, Arnoczky SP, Dodds J, Ross H. The effect of drilling and soft tissue grafting across open growth plates. A histologic study. Am J Sports Med. 1995;23:431-5.

20. Shea KG, Apel PJ, Pfeiffer RP, Traughber PD. The anatomy of the proximal tibia in pediatric and adolescent patients: implications for ACL reconstruction and prevention of physeal arrest. Knee Surg Sports Traumatol Arthrosc. 2007;15:320-7.

21. Anderson AF. Transepiphyseal replacement of the anterior cruciate ligament using quadruple hamstring grafts in skeletally immature patients. J Bone Joint Surg Am. 2004;86A Suppl 1:201-9.

22. Gebhard F, Ellermann A, Hoffmann F, Jaeger JH, Friederich NF. Multicenter-study of operative treatment 
of intraligamentous tears of the anterior cruciate ligament in children and adolescents: comparison of four different techniques. Knee Surg Sports Traumatol Arthrosc. 2006;14:797-803.

23. Schachter AK, Rokito AS. ACL injuries in the skeletally immature patient. Orthopedics. 2007;30:365-70.

24. Bonnard C, Fournier J, Babusiaux D, Planchenault M, Bergerault F, de Courtivron B. Physeal-sparing reconstruction of anterior cruciate ligament tears in children: results of 57 cases using patellar tendon. J Bone Joint Surg Br. 2011;93:542-7.

25. Lyman S, Koulouvaris P, Sherman S, Do H, Mandl LA, Marx RG. Epidemiology of anterior cruciate ligament reconstruction: trends, readmissions, and subsequent knee surgery. J Bone Joint Surg Am. 2009;91:2321-8.

26. Streich NA, Barie A, Gotterbarm T, Keil M, Schmitt H.
Transphyseal reconstruction of the anterior cruciate ligament in prepubescent athletes. Knee Surg Sports Traumatol Arthrosc. 2010;18:1481-6.

27. Frosch KH, Stengel D, Brodhun T, Stietencron I, Holsten D, Jung C, Reister D, Voigt C, Niemeyer P, Maier M, Hertel P, Jagodzinski M, Lill H. Outcomes and risks of operative treatment of rupture of the anterior cruciate ligament in children and adolescents. Arthroscopy. 2010;26:1539-50.

28. Paley D, Pfeil J. Principles of deformity correction around the knee. Orthopade. 2000;29:18-38.

29. Briggs KK, Steadman JR, Hay CJ, Hines SL. Lysholm score and Tegner activity level in individuals with normal knees. Am J Sports Med. 2009;37:898-901.

30. Kim SJ, Kim TE, Lee DH, Oh KS. Anterior cruciate ligament reconstruction in patients who have excessive joint laxity. J Bone Joint Surg Am. 2008;90:735-41. 